Paper presented at the Workshop on Space Charge Physics in High Intensity Hadron Rings, sponsored by Brookhaven National Laboratory, May 4-7, 1998

$$
\text { CONF-980567-- }
$$

\title{
Nonlinear Self Consistent High Resolution Beam Halo Algorithm in Homomorphic and Weakly Chaotic Systems
}

\author{
J. H. Whealton, R. J. Raridon, D. K. Olsen, J. D. Galambos, J. A. Holmes
}

Oak Ridge National Laboratory

Engineering Technology, Physics, and Computational Physics and Engineering Divisions

Prepared by the

Oak Ridge National Laboratory

Oak Ridge, Tennessee 37831-8088

Managed by

Lockheed Martin Energy Research Corp.

for the

U.S. Department of Energy

Under contract DE-AC05-960R22464
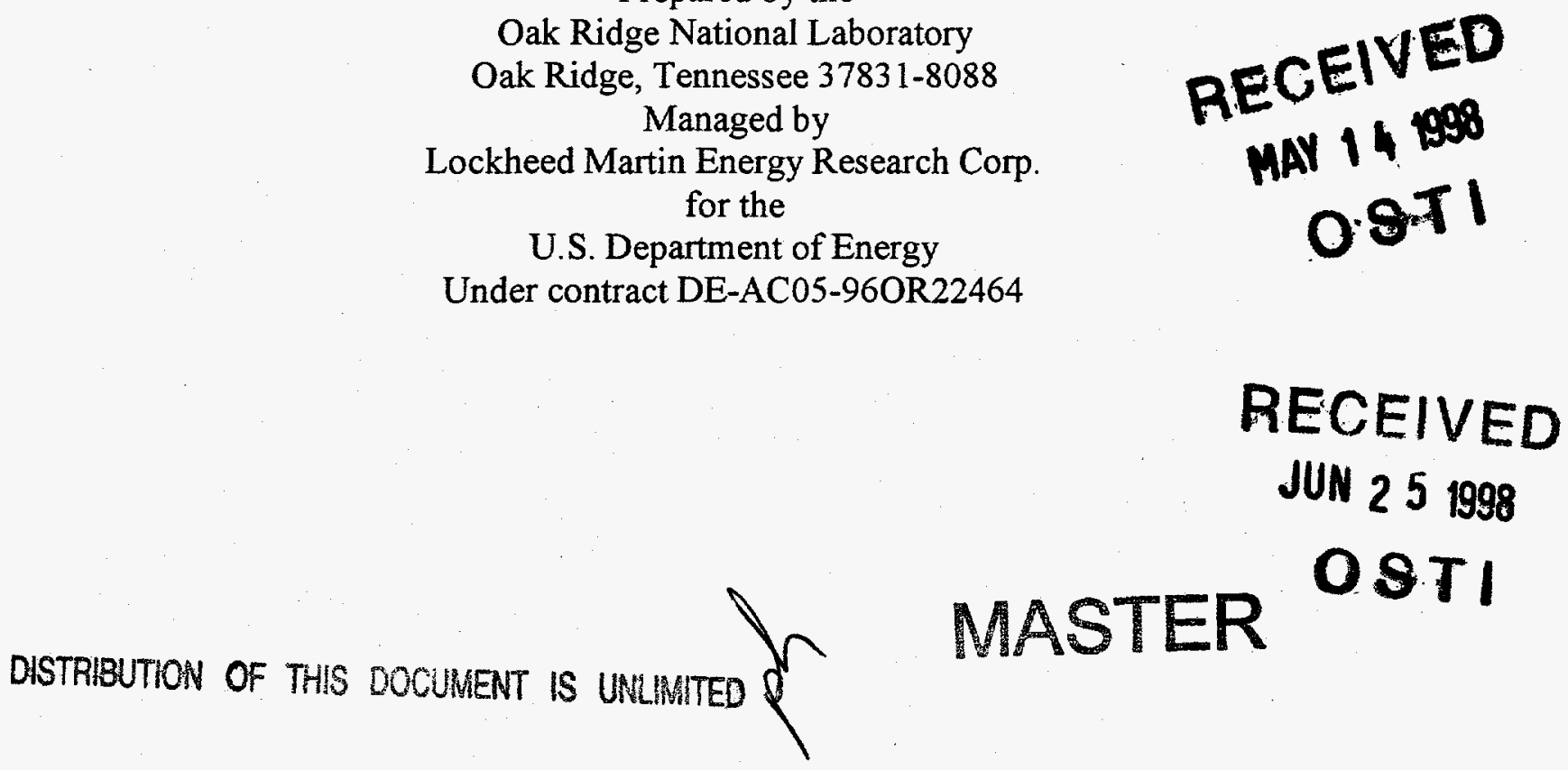

The submitted manuscript has been authored by a contractor of the U.S. Government under contract No. DE-AC05-960R22464. Accordingly, the U.S. Government retains a nonexclusive, royalty-free license to publish or reproduce the published form of this contribution, or allow others to do so, for U.S. Government purposes." 


\section{DISCLAIMER}

This report was prepared as an account of work sponsored by an agency of the United States Government. Neither the United States Government nor any agency thereof, nor any of their employees, makes any warranty, express or implied, or assumes any legal liability or responsibility for the accuracy, completeness, or usefulness of any information, apparatus, product, or process disclosed, or represents that its use would not infringe privately owned rights. Reference herein to any specific commercial product, process, or service by trade name, trademark, manufacturer, or otherwise does not necessarily constitute or imply its endorsement, recommendation, or favoring by the United States Government or any agency thereof. The views and opinions of authors expressed herein do not necessarily state or reflect those of the United States Government or any agency thereof. 


\section{DISCLAIMER}

Portions of this document may be illegible electronic image products. Images are produced from the best available original document. 


\section{ABSTRACT}

A technique is described which enables high resolution of halo in beam dynamic studies by direct simulation. The method consists in first solving the beam dynamics problem using coarse initial data. The regions of the initial data, which result in beam halo, or extremums in phase space, are identified. The dynamics are resolved by continuing the calculation using initial data points slightly offset from those that result in halo formation, thus filling in the halo structure. The solution is repeated with appropriate scaling of such things as charge per orbit etc. This process may be continued indefinitely. The method can also shed some light on the halo generation in weakly chaotic systems. The scheme is essentially different from the $\Delta \mathrm{f}$ method in that no assumption is made about $\mathrm{f}_{0}$. As an example, a bifurcation in a non-trivial space charge dominated homomorphic problem is resolved self-consistently using minor computational resources, rather than having to perform the calculation for 250 trillion effective particles.

\section{INTRODUCTION}

Recent accelerator systems require a very low beam-wall interception (as low as 1 part in $10^{6}$ ). Modeling the beam dynamics including significant statistical sample in the halo would require considering on the order of a billion trajectories. In certain cases, which are not chaotic or weakly chaotic, a technique may be used which requires substantially fewer resources than the brute force approach.

The idea is easily understandable by reference to a specific example as shown in Fig. 1. Here is modeled an ion extraction from a plasma. The following Vlasov-Poison equations

$$
\begin{gathered}
\nabla^{2} \varphi(\mathbf{r}, \mathbf{t})=\int \mathrm{f}(\mathbf{r}, \mathbf{v}, \mathbf{t}) \mathrm{d} \mathbf{v}-\exp [-\varphi(\mathbf{r}, \mathrm{t})] \\
\frac{\partial \mathrm{f}(\mathbf{r}, \mathbf{v}, \mathbf{t})}{\partial \mathbf{t}}+\mathbf{v} \bullet \nabla \mathbf{f}(\mathbf{r}, \mathbf{v}, \mathrm{t})+\{\mathbf{v} \times \mathbf{B}-\nabla \varphi\} \bullet \nabla_{\mathbf{v}} \mathrm{f}(\mathbf{r}, \mathbf{v}, \mathbf{t})=0
\end{gathered}
$$


provide an adequate description. Plasma electron density is described by a Boltzman distribution. Electrostatic equipotentials are shown in this 2-D example by dashed lines. Solid lines show ion trajectories (only on the top half of the figure). The extraction sheath, found selfconsistently, is shown by the high field region in the middle of the figure. (Plasma electrons are abundant on the left side of this sheath and absent on the right side.) One electrode is shown in the middle of the figure on the top and bottom. The beam goes off the right side of the figure. The occupation in phase space of the exiting beam is shown in Fig. 2. Of particular interest are the beam aberrations shown in Fig. 2 due to the nonlinear transverse forces in the proximity of the electrode (shown in Fig. 1).

The scheme is basically to take those trajectories which are in the outer reaches of phase space, trace them back to their starting position (on the left side of Fig. 1) and then create, say ten trajectories, in the phase space neighborhood of the ten most aberrated trajectories. This is a very simple thing to do in the example illustrated in Fig. 1. First, the 10 most aberrational trajectories are replaced by 100 trajectories with a space charge weight of one-tenth the original. The self-consistent solution is shown in Fig. 3 (corresponding to Fig. 1) and Fig. 4 (corresponding to Fig. 3). This is denoted as the second major iteration. A comparison of Fig. 3 with Fig. 1 shows exactly what trajectories were selected. A comparison of Fig. 4 with Fig. 2 shows the corresponding region in phase space that was refined. Continuation of the process is done by again selecting the ten most aberrated trajectories in Figs. 3 and 4, replacing each of them with ten trajectories with a weighting of one-tenth of the replaced trajectory. This is the third major iteration. The result after 13 major iterations is shown in Figs. 5 and 6 . As can be seen in Table 1, after 13 major iterations, only 10,306 cumulative trajectories were calculated, but if the resolution were uniform over all of the phase space 252 trillion trajectories would have 
been calculated - a saving of a factor of 25 billion in computation time. If all of these other 252 trillion trajectories were actually computed, the result would simply fill in the line in the central part of the phase space diagram - Fig. 6 . While this factor may not be easily realizable in more complex problems with nontrivial occupation of $6 \mathrm{D}$-phase space, large factors of resource savings in self-consistent halo calculations are still possible.

For non-chaotic, completely deterministic systems, there is no limit to the savings possible. For weakly chaotic systems, there will be a limit to the resolution obtainable, but information will be found on these chaotic regions of the phase space. For highly chaotic systems, the method will not be reliable. 


\begin{tabular}{|c|c|c|}
\hline $\begin{array}{l}\text { Major } \\
\text { Iteration }\end{array}$ & Actual & Apparent \\
\hline 1 & $126 \times 2=252$ & 252 \\
\hline 2 & $\begin{array}{l}252-10+100=342 \\
\Sigma 342+252=594\end{array}$ & 2520 \\
\hline 3 & $\begin{aligned} 342-10+100 & =432 \\
\Sigma & =1026\end{aligned}$ & 25,200 \\
\hline 4 & $\begin{aligned} 432-10+100 & =522 \\
\Sigma & =1548\end{aligned}$ & 252,000 \\
\hline 5 & $\begin{aligned} 522-10+100 & =612 \\
\Sigma & =2170\end{aligned}$ & $2,520,000 \quad(\mathbf{M})$ \\
\hline 6 & $\begin{aligned} 612-10+100 & =702 \\
\Sigma & =2872\end{aligned}$ & $25,200,000$ \\
\hline 7 & $\begin{aligned} 702-10+100 & =792 \\
\Sigma & =3664\end{aligned}$ & $257,000,000$ \\
\hline 8 & $\begin{aligned} 792-10+100 & =882 \\
\Sigma & =4546\end{aligned}$ & $2,520,000,000$ \\
\hline 9 & $\begin{aligned} 882-10+100 & =972 \\
\Sigma & =5518\end{aligned}$ & $25,200,000,000$ \\
\hline 10 & $\begin{aligned} 972-10+100 & =1062 \\
\Sigma & =6580\end{aligned}$ & $252,000,000,000$ \\
\hline 11 & $\begin{aligned} 1062-10+100 & =1152 \\
\Sigma & =7732\end{aligned}$ & $2,520,000,000,000$ \\
\hline 12 & 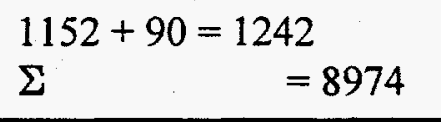 & $25.2 \mathrm{~T}$ \\
\hline 13 & $\begin{array}{l}1242+90=1332 \\
\Sigma \quad=10306\end{array}$ & 252.T \\
\hline
\end{tabular}




\section{Figure Legends}

Figure 1. Ion orbits (solid lines starting at the left from inside the plasma) are accelerated by externally applied electric fields (the equipotentials indicated by dashed lines). The selfconsistent plasma sheath is shown by the high field region (close spacing of equipotentials) shown. An electrode (constant equipotential) is shown at the middle top and bottom. The ion beam, which exists on the right, has a halo produced by aberrations caused by nonlinear fields near the electrode.

Figure 2. Phase space plot of the beam existing on the right hand side, Fig. 1, the transverse distance is the horizontal axis and the transverse velocity is the vertical axis. The points with large transverse speeds represent the aberrations (halo) of the beam, in this case.

Figure 3. Same as Fig. 1 except the most aberrated 10 orbits are replaced by several more (100) for enhanced resolution.

Figure 4. Same as Fig. 2 but now refers to the exiting beam of Fig. 3.

Figure 5. Same as Fig. 3 but the process has been continued 11 more times giving much greater resolution near the region of aberrations.

Figure 6. Same as Fig. 4, but now referring to Fig. 5. Now the aberrations are fully resolved. 


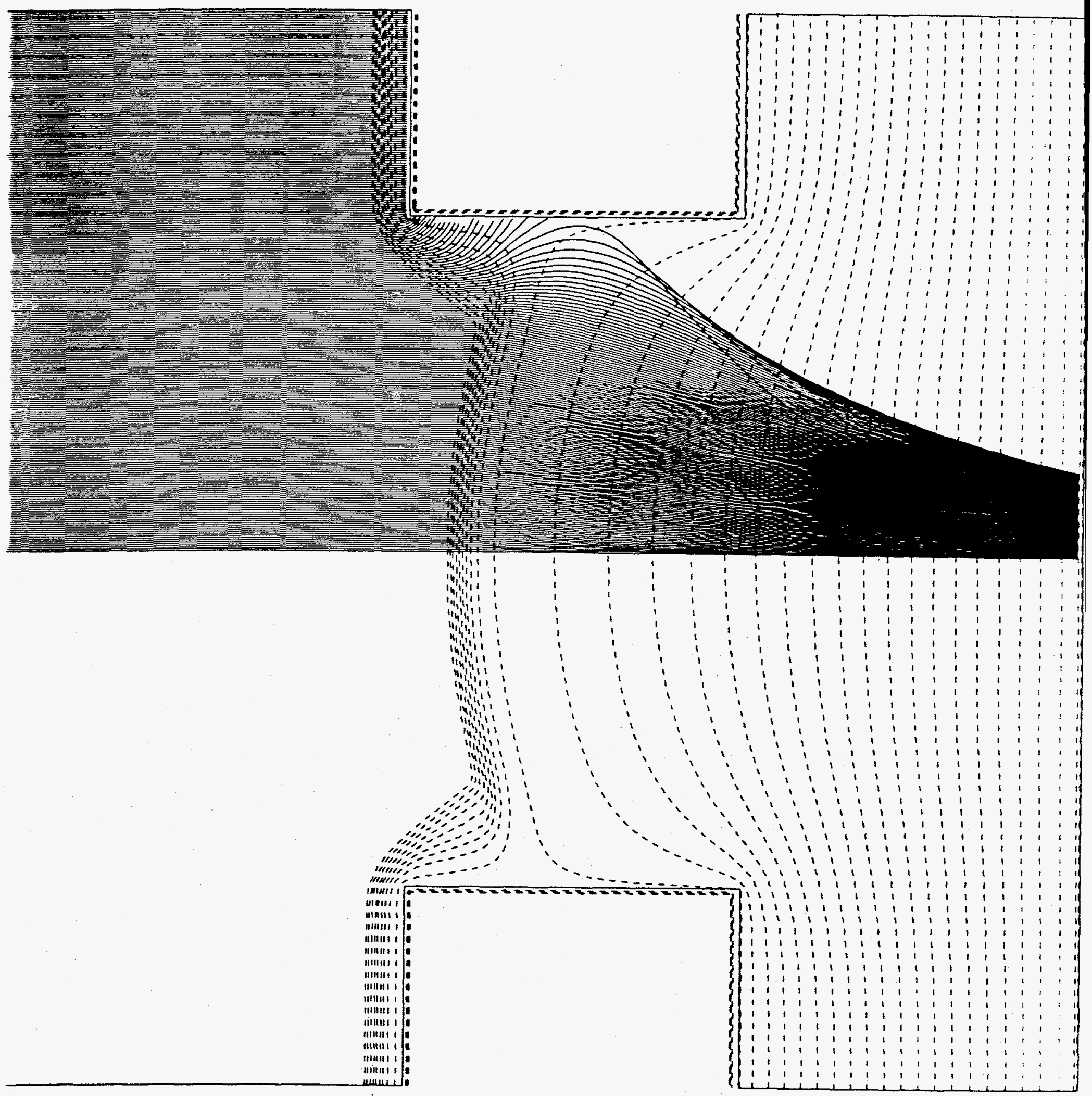




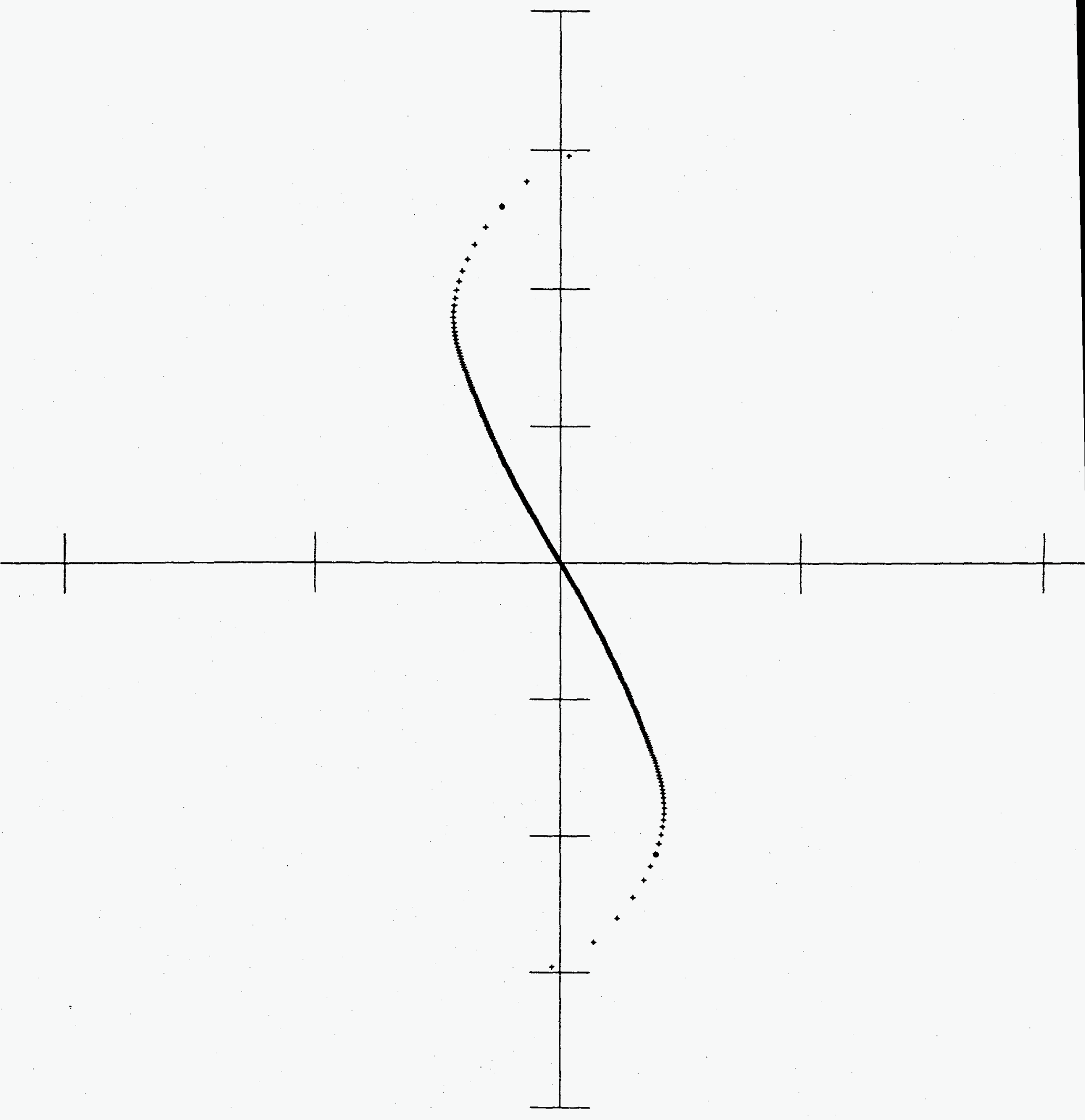




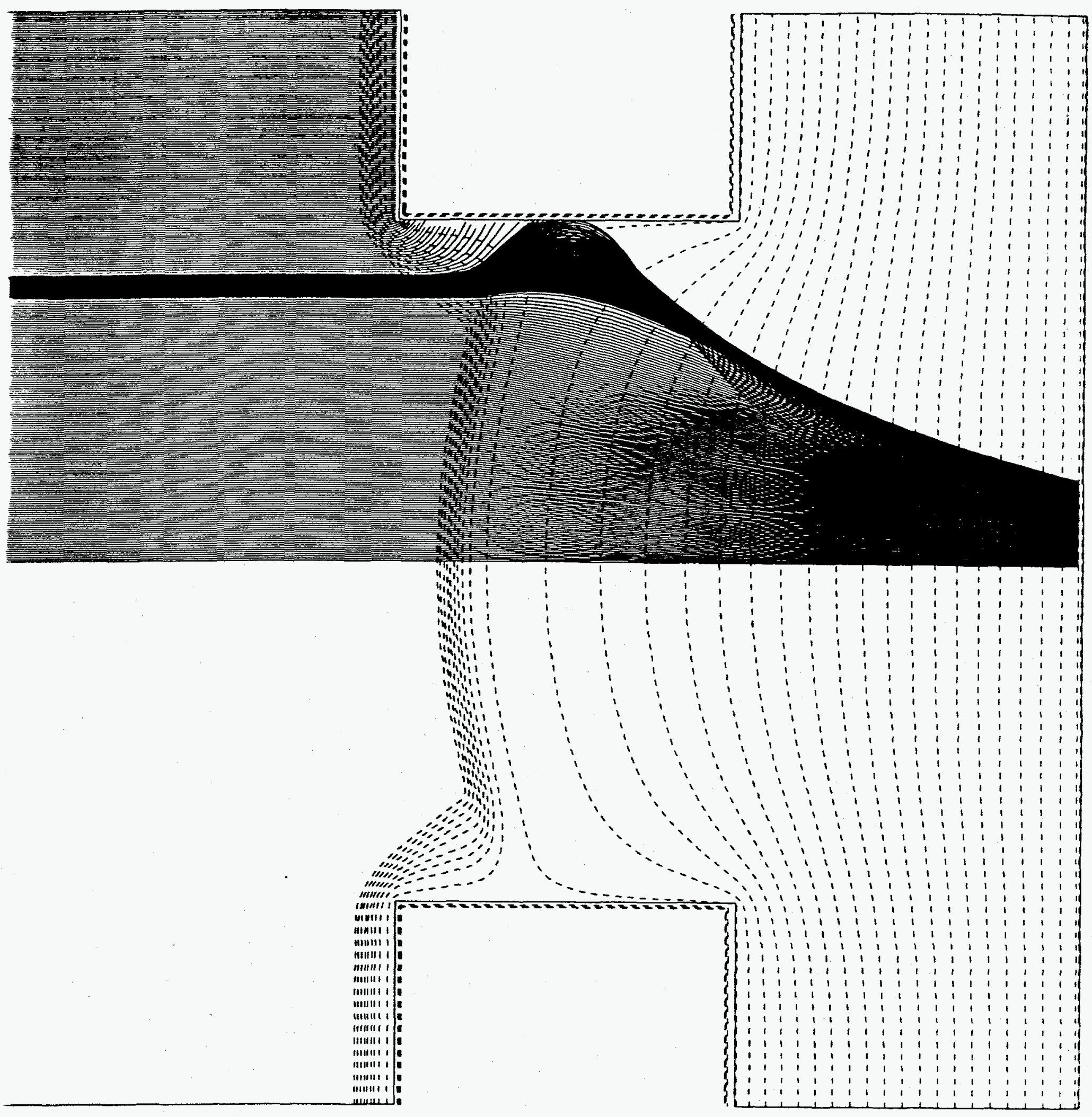




$$
\int
$$




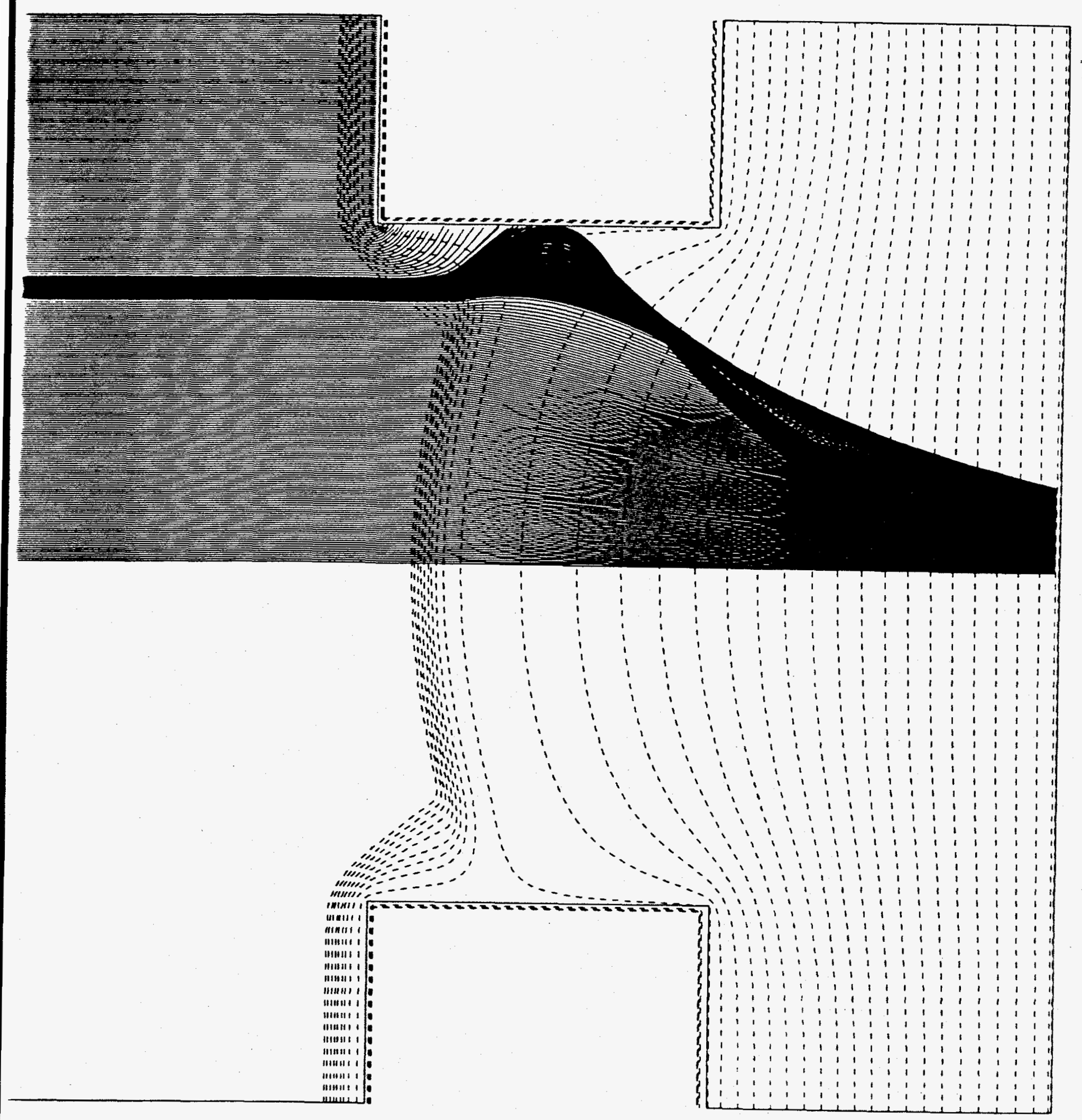




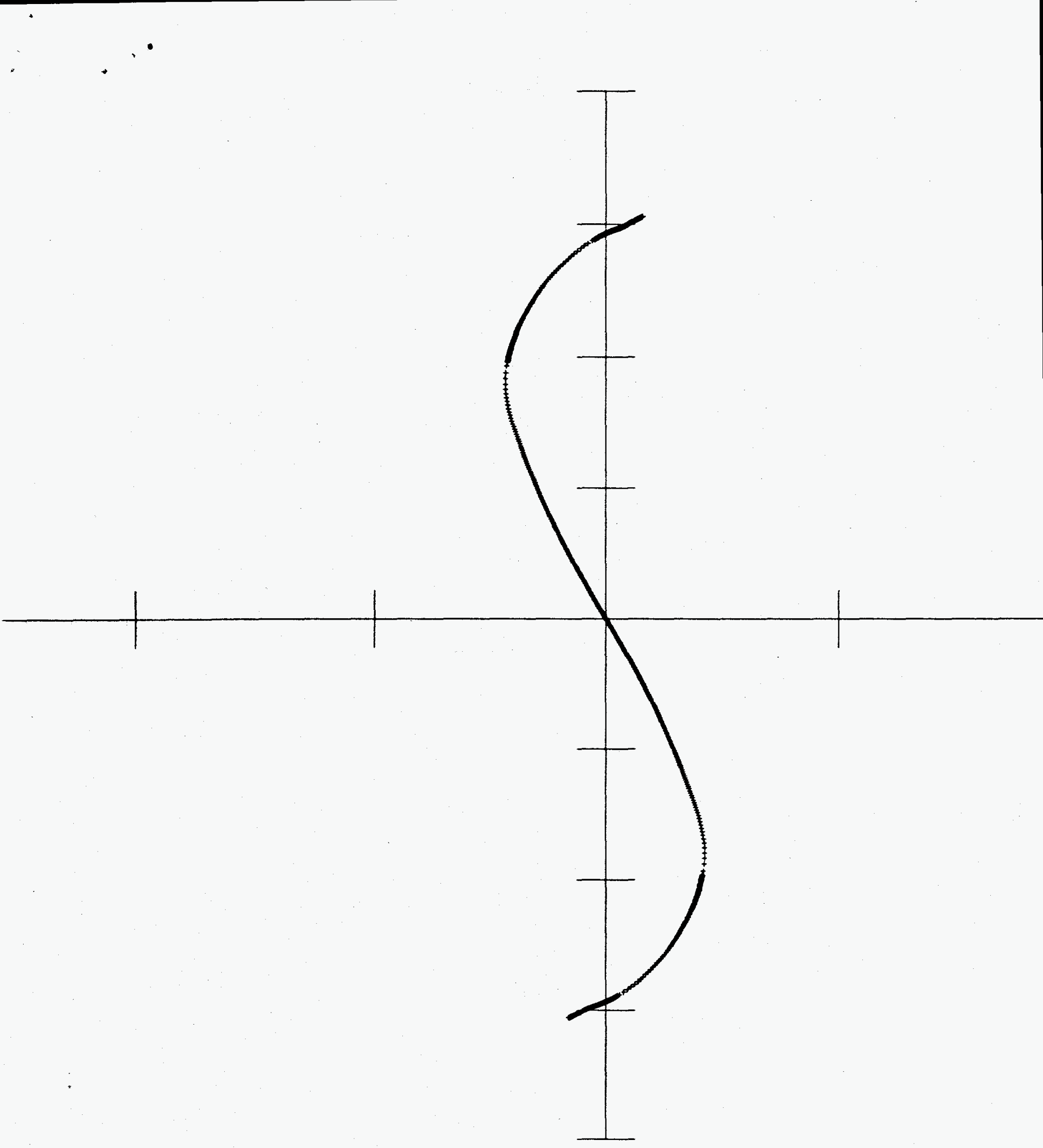

